OPEN ACCESS

Edited by:

Billy Sperlich,

Universität Würzburg, Germany

Reviewed by:

José González-Alonso,

Brunel University London,

United Kingdom

Hsiaotung Yang,

University of Missouri, United States

${ }^{*}$ Correspondence:

A. Yaël Nossent

a.y.nossent@/umc.n

Specialty section:

This article was submitted to

Exercise Physiology,

a section of the journal

Frontiers in Physiology

Received: 11 May 2018

Accepted: 30 July 2018

Published: 22 August 2018

Citation:

Håkansson KEJ, Sollie O, Simons KH, Quax PHA, Jensen J and Nossent AY

(2018) Circulating Small Non-coding

RNAs as Biomarkers for Recovery

After Exhaustive or Repetitive

Exercise. Front. Physiol. 9:1136.

doi: 10.3389/fphys.2018.01136

\section{Circulating Small Non-coding RNAs as Biomarkers for Recovery After Exhaustive or Repetitive Exercise}

\author{
Kjell E. J. Håkansson 1,2, Ove Sollie ${ }^{3}$, Karin H. Simons ${ }^{1,2}$, Paul H. A. Quax ${ }^{1,2}$, \\ Jørgen Jensen ${ }^{3}$ and $A$. Yaël Nossent ${ }^{1,2,4 *}$
}

${ }^{1}$ Department of Vascular Surgery, Leiden University Medical Center, Leiden, Netherlands, ${ }^{2}$ Einthoven Laboratory for Experimental Vascular Medicine, Leiden University Medical Center, Leiden, Netherlands, ${ }^{3}$ Department of Physical Performance, Norwegian School of Sport Sciences, Oslo, Norway, ${ }^{4}$ Ludwig Boltzmann Cluster for Cardiovascular Research, Vienna, Austria

Circulating microRNAs have proven to be reliable biomarkers, due to their high stability, both in vivo in the circulation, and ex vivo during sample preparation and storage. Small nucleolar RNAs (snoRNAs) are a different type of small non-coding RNAs that can also be reliably measured in plasma, but have only been studied sporadically. In this study, we aimed to identify RNA-biomarkers that can distinguish between different exercise regimes and that entail clues about muscle repair and recovery after prolonged exhaustive endurance exercise. We compared plasma microRNA profiles between two cohorts of elite cyclists, subjected to two different types of exercise regimes, as well as a cohort of patients with peripheral artery disease (PAD) that were scheduled to undergo lower limb amputation, due to critical limb ischemia. In elite athletes, muscle tissue recovers quickly even after exhaustive exercise, whereas in PAD patients, recovery is completely impaired. Furthermore, we measured levels of a specific group of snoRNAs in the plasma of both elite cyclists and PAD patients. Using a multiplex qPCR screening, we detected a total of 179 microRNAs overall, of which, on average, 161 microRNAs were detected per sample. However, only 30 microRNAs were consistently expressed in all samples. Of these, two microRNAs, miR-29a-3p and miR193a-5p, that responded differently two different types of exercise, namely exhaustive exercise and non-exhaustive endurance exercise. Using individual rt/qPCR, we also identified a snoRNA, SNORD114.1, which was significantly upregulated in plasma in response to endurance exercise. Furthermore, two microRNAs, miR-29a-3p and miR-495-3p, were significantly differentially expressed in athletes compared to PAD patients, but only following exercise. We suggest that these two microRNAs could function as markers of impaired muscle repair and recovery. In conclusion, microRNAs miR-29a-3p and miR-193a-5p may help us distinguish between repeated exhaustive and non-exhaustive endurance exercise. MicroRNA miR-29a-3p, as well as miR-495-3p, may further mark impaired muscle recovery in patients with severe critical limb ischemia. Furthermore, we showed for the first time that a circulating snoRNA, SNORD114.1, is regulated in response to exercise and may be used as biomarker.

Keywords: snoRNA, exercise physiology, biomarker, microRNA, peripheral artery disease 


\section{INTRODUCTION}

Regular exercise at moderate intensity has clear benefits for general health, overall fitness, quality of life and importantly, cardiovascular physiology. Exercise at elite level however, is far more demanding of the body and muscles. During high intensity endurance exercise, muscle glycogen is the major energy substrate and metabolic stress develops. Moreover, exercise triggers an increase in cytokine production, attraction of leukocytes, upregulation of pro-angiogenic stimuli and an overall pro-inflammatory state (Ulven et al., 2015; Langleite et al., 2016). In healthy individuals however, during restitution and repletion of glycogen levels, the inflammation is dampened and over time, muscle function improves.

In patients with peripheral artery disease (PAD), poor circulation leads to a chronic ischemia and low levels of nutrients in the affected limb(s). PAD is caused by chronic accumulation of atherosclerotic lesions in the arteries of the limbs, generally of the leg (Abul-Khoudoud, 2006). Besides lifestyle changes, such as cessation of smoking and increased moderate exercise, and management of risk factors, such as cholesterol lowering, there are no therapeutics available that effectively treat PAD. Patients with severe PAD are at risk of developing critical limb ischemia, defined by cramp-like pain even at rest. Unless blood flow is recovered via physiological neovascularization (i.e., arteriogenesis and angiogenesis), or via surgical revascularization (i.e., stenting or bypass surgery), the pro-inflammatory state becomes permanent and instead of recovery, muscle tissue will start to degrade and ultimately become necrotic. In such severe cases, the only remaining treatment option to prevent sepsis and death is amputation of the affected limb (Germani et al., 2009).

Both in exercise physiology and in PAD pathology, researchers have been trying to identify reliable biomarkers to follow muscle repair and recovery. In exercise physiology, such biomarkers could be used to improve training and recovery schedules to optimize competitive performance. Whereas in PAD, such biomarkers may assist vascular surgeons and PAD patients in their joint decision-making before surgical intervention, it being revascularization or even amputation. Although the respective perspectives are very different, potentially, both fields of research could stand to benefit from each other.

In recent years, circulating microRNAs have proven to be highly reliable biomarkers (Mitchell et al., 2008; Hakimzadeh et al., 2015). MicroRNAs are small, non-coding RNA molecules that inhibit the translation of messenger RNAs by binding to their $3^{\prime}$ untranslated regions (Bartel, 2004). Although microRNAs exert their function intracellularly, microRNAs can also be released into the circulation, either passively upon tissue damage, or actively bound to lipid particles such as HDL, or in microvesicles or exosomes. Microvesicles are also released upon exercise (Wilhelm et al., 2016; Whitham et al., 2018). While extracellular RNA is usually rapidly digested by endogenous RNases, the short length of microRNAs (around 20 nucleotides) protects them from most RNase degradation and gives them relative extracellular stability (Mitchell et al., 2008). Furthermore, microRNA are often highly tissue- or pathway-specificity. Within muscle physiology, muscle-specific microRNAs (myomiRs) such as miR-1 miR-133a, miR-133b, and miR-206 have indeed been detected in the circulation after exercise (Horak et al., 2016). Other studies have shown that non-myomiRs, including the hepatic miR-122, are also increased in the circulation during exercise trials (Cui et al., 2015).

Research into other types of non-coding RNAs, including small nucleolar RNA (snoRNA) has increased as well over the past few years, but little is known about their role in exercise and muscle physiology. Similar to microRNAs, snoRNAs are small, highly conserved RNAs. Their canonical function is to stabilize other RNA species, either through $2^{\prime} O$-ribose-methylation (Kiss, 2001), or through pseudouridylation. SnoRNAs have been shown to be reliably measurable in plasma, making them promising candidates for biomarkers, much like microRNAs (Liao et al., 2010).

Besides increased extracellular stability in vivo, small noncoding RNAs have also proven to be highly stable ex vivo, resisting degradation due to poor sample handling, repetitive freezethawing and long-term storage. This remarkable stability may give small non-coding RNAs a further advantage as biomarkers (Peiro-Chova et al., 2013).

In this study, we have performed a screening for circulating microRNAs regulated after exhaustive endurance exercise in a cohort of elite cyclists. Regulated microRNAs were then confirmed and further investigated in a second cohort of elite cyclists performing a $60 \mathrm{~min}$ time trial at highest possible intensity. Furthermore, plasma microRNAs were compared between an athlete cohort and a cohort of PAD patients hospitalized for critical limb ischemia, scheduled for lower limb amputation. Finally, we measured levels of a specific group of snoRNAs in the plasma of both elite cyclists and PAD patients.

By comparing the effects of muscle exhaustion on circulating non-coding RNAs between these two extreme ends of the spectrum, from highly trained elite-cyclists to patients in the endstage of PAD, we aimed identify strong and reliable biomarkers for muscle repair and recovery.

\section{MATERIALS AND METHODS}

A schematic overview of our study set-up is presented in Figure 1.

\section{Exhaustive Endurance Exercise (EEE) Cohort}

\section{Subjects}

Eight male elite endurance cyclists (mean $\pm \mathrm{SD}$; age: $22.9 \pm 1.2$ years; height: $182 \pm 2 \mathrm{~cm}$; body mass: $79.5 \pm 3 \mathrm{~kg}$ ) were recruited for our cohort. All subjects had competitive experience at national or international level and trained, $16 \pm 1 \mathrm{~h}$ weekly, of which $12 \pm 2 \mathrm{~h}$ was cycling.

This study was carried out in accordance with the recommendations of Guidelines for Human Exercise Trials, Norwegian School of Sport Sciences' Ethics Committee. The protocol was reviewed by Regional Ethic Committee of Norway (2011/1298) with the decision that the research project was outside the Act on Medical Health Research, confirmed in letter of exemption (2011/1298; IRB Reference No. IRB00006245). The 

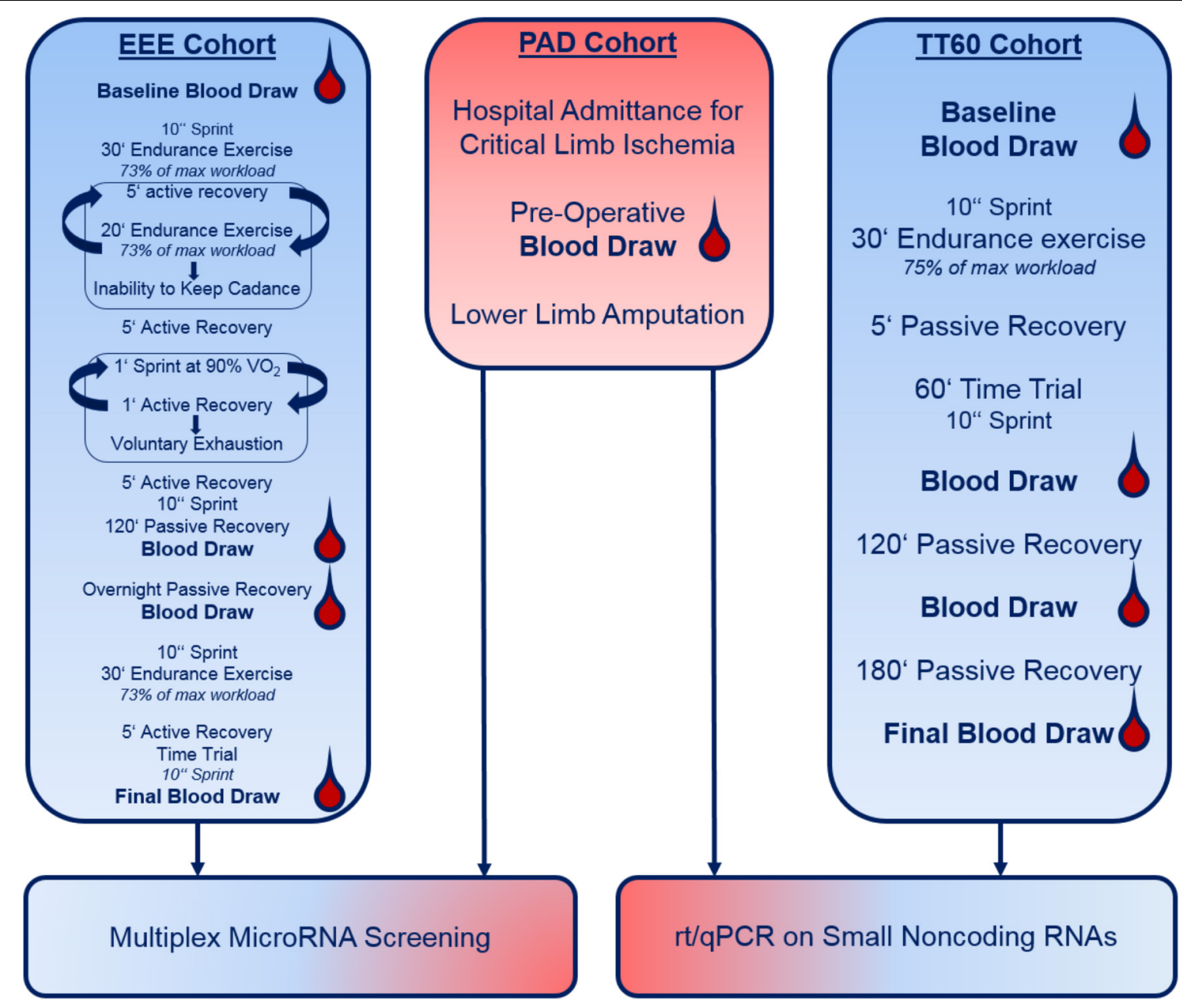

FIGURE 1 | Schematic representation of the study cohorts and set-up. Please note that details on warming-up, etc. are not provided (see section "Materials and Methods").

study was conducted according to the Declaration of Helsinki and participants gave their informed written consent after oral and written information about the study.

\section{Study Set-Up}

Before trial start, subjects completed a familiarization session with a self-paced warm up and a possibility for subjects to adjust the ergometer (Lode Excalibur Sport, Groningen, Netherlands) geometry to individual preferences, as previously described (Rustad et al., 2016). Linear regression was used to identify the workload corresponding to $73 \%$ of $\mathrm{VO}_{2 \max }\left(\mathrm{W}_{73 \%}\right)$.

On the first trial day an exhaustive exercise session was performed. The session started with a warm up as described previously (Rustad et al., 2016). After 5 min of recovery, subjects performed a $10 \mathrm{~s}$ all-out effort sprint followed by another $5 \mathrm{~min}$ of recovery. The exhaustive exercise session started with $30 \mathrm{~min}$ at $\mathrm{W}_{73 \%}$, followed by another $5 \mathrm{~min}$ of recovery. Subsequent intervals of $20 \mathrm{~min}$ at $\mathrm{W}_{73 \%}$ with $5 \mathrm{~min}$ of rest between intervals were performed until subjects were unable to maintain predetermined cadence \pm 5RPM. After exhaustion, athletes were given $5 \mathrm{~min}$ of recovery, followed by repeated intervals of $60 \mathrm{~s}$ at $90 \% \mathrm{VO}_{2 \max }$-sprints with $60 \mathrm{~s}$ of rest between sprints until voluntary exhaustion. Blood samples were drawn at baseline and at $120 \mathrm{~min}$ after the exercise session.

On the second day, roughly $18 \mathrm{~h}$ after finishing the first exhaustive exercise session, subjects completed the same warm up as on the previous day. After warm up, $5 \mathrm{~min}$ of recovery was followed by a $10 \mathrm{~s}$ sprint, followed by $30 \mathrm{~min}$ cycling at $\mathrm{W}_{73 \%}$. After 5 min rest, a time test was performed with a predetermined amount of work [Work $(\mathrm{kJ})=$ Power at $\mathrm{VO}_{2 \max }$ $(\mathrm{W}){ }^{*} 1800 \mathrm{~s}$. Initial workload was set to $\mathrm{W}_{73 \%}$, but the athletes were free to adjust workload to personal preference. Five minutes after the time-trial participants performed a $10 \mathrm{~s}$ all-out effort sprint. Blood was drawn before warm up and immediately after completion of the exercise session.

\section{Sample Collection}

Blood was drawn from the antecubital vein using a Teflon catheter (18GA, BD Venflon Pro, Franklin Lakes, NJ, United States) into EDTA-coated tubes and centrifuged 
$(2500 \mathrm{~g}, 10 \mathrm{~min})$ at $4^{\circ} \mathrm{C}$. Plasma was transferred to $1.5 \mathrm{ml}$ tubes and stored at $-80^{\circ} \mathrm{C}$ until analysis.

\section{Peripheral Artery Disease (PAD) Cohort - Ampubase \\ Subjects}

Sixteen patients ( $87.5 \%$ male; age: $69 \pm 13$ years) from the Leiden University Medical Center biobank Ampubase were included in our study. All patients were scheduled for lower limb amputation, either below or above the knee joint as a consequence of critical limb ischemia. Inclusion criteria for the biobank were a minimum age of 18 years and lower limb amputation, excluding ankle, foot, or toe amputations. Exclusion criteria were confirmed or suspected malignancy and inability to give informed consent.

The study was approved by the Medical Ethics Committee of the Leiden University Medical Center (Protocol No. P12.265) and written informed consent was obtained from all participants.

\section{Sample Collection}

Blood samples were collected pre-operatively. Sample collection followed standard hospital procedures. Blood was collected in citrate coated tubes and immediately put on ice. Samples were processed within $30 \mathrm{~min}$ of collection and were centrifuged for $10 \mathrm{~min}$ at $2800 \mathrm{~g}$ at $4^{\circ} \mathrm{C}$. Plasma was then aliquoted and the plasma closest to the buffy coat was discarded. Aliquots were stored at $-80^{\circ} \mathrm{C}$ until analysis.

\section{Time Trial (TT60) Cohort Study Population}

Thirteen highly trained, male endurance cyclists (mean $\pm \mathrm{SD}$; age: $27.7 \pm 1.2$ years; height: $184 \pm 1 \mathrm{~cm}$; body mass: $77.2 \pm 1.1 \mathrm{~kg}$ ) were recruited for this study. All subjects had competitive experience at national or international level and trained for $11.5 \pm 1.2 \mathrm{~h}$ weekly, of which $8.8 \pm 1.4 \mathrm{~h}$ was cycling. Due to sample depletion, only 10 athletes with complete sets of samples were included in our analyses.

All subjects were informed of any potential risks before acquiring their written informed consent. The project was approved by Regional Ethics Committee (2013/1528) of Norway, and performed according to the Declaration of Helsinki.

\section{Study Set-Up}

The initial pre-trial testing, analysis and equipment used in the second cohort was identical to the EEE cohort, described above.

On the day of the trial, subjects started with a standardized warm up of 5-2 min of $50,55,60$, and $75 \%$ of the pre-trial $\mathrm{VO}_{2 \max }$, respectively. The exercise session was started with a $10 \mathrm{~s}$ all-out sprint immediately followed by $4 \mathrm{~min}$ of active (100W) or passive restitution. After initial restitution, participants cycled $30 \mathrm{~min}$ at $\mathrm{W}_{73 \%}$, followed by a $60 \mathrm{~min}$ time trial separated by 5 min of restitution. The session ended with another 10 se all-out sprint.

Blood samples were drawn before exercise, immediately after exercise completion as well as after 2 and $5 \mathrm{~h}$ of restitution.

\section{Sample Collection}

Blood was drawn from the antecubital vein using a Teflon catheter (18GA, BD Venflon Pro, Franklin Lakes, NJ, United States) into EDTA-coated tubes. Samples were stored on ice until centrifugation $\left(10 \mathrm{~min}, 2500 \mathrm{~g}\right.$ ) at $4^{\circ} \mathrm{C}$, after which plasma was aliquoted and stored at $-80^{\circ} \mathrm{C}$ until subsequent analysis.

\section{RNA Isolation and Quantification}

Plasma samples from five male EEE Cohort participants and five male Ampubase Cohort participants, were sent to Exiqon Services, Exiqon A/S (Vedbæk, Denmark) for RNA isolation, reverse transcription and Multiplex Circulating MicroRNA qPCR Panel for microRNA expression analysis, using their in-house products and protocols. Samples were selected on relative homogeneity in age and other physical parameters.

Total RNA from $100 \mu \mathrm{L}$ of plasma was extracted using the miRCURY RNA Isolation Kit - Biofluids (Exiqon, Vedbæk, Denmark), including rDNase treatment to prevent DNA contamination. RNA was stored until further analysis at $-80^{\circ} \mathrm{C}$.

RNA was reverse transcribed using the miRCURY LNA Universal microRNA PCR, Polyadenylation and cDNA Synthesis Kit. Each microRNA was assayed by qPCR using microRNA Ready-to-Use PCR, Serum/Plasma Focus panel using ExiLENT SYBR Green Master Mix. Negative controls were included as master mix without cDNA template. Amplification was performed using the LightCycler 480 Real-Time PCR System (Roche Molecular Diagnostics, Pleasanton, CA, United States). Amplification curves were analyzed using the Roche LC software, both for determination of $\mathrm{Cq}$ and for melt curve analysis.

Amplification efficiency was calculated using Exiqon's inhouse algorithms similar to the LinReg software. Melting curves and temperatures of all assays were within specifications of the assays. All measurements with five Cqs less than the negative control and with a $\mathrm{Cq}<37$ were included in the analysis. Data that did not meet these criteria were omitted from further analyses. All data were normalized to the average of all assays in all samples (average $\mathrm{Cq}$ - assay $\mathrm{Cq}$ ).

For snoRNA and confirmatory microRNA analyses, total RNA was isolated from all available plasma samples using TRIzolLS Reagent, according to the Manufacturer's protocol (Thermo Fisher, Waltham, MA, United States).

For snoRNAs analyses, RNA was reverse transcribed using 'High-capacity RNA to cDNA kit' (Thermo Fisher, Waltham, MA, United States) and quantified by RT-qPCR using SybrGreen reagent (Qiagen, Venlo, Netherlands) on the VIIa7 (Thermo Fisher, Waltham, MA, United States). All qPCR reactions were performed in technical triplicates. The Coefficient of Variance $\left(\mathrm{C}_{v}=\Sigma / \mu\right)$ was calculated and triplicates resulting in a $\mathrm{C}_{v}>0.02$ were excluded. SnoRNA expression was normalized to U6.

For confirmatory microRNA quantification, RNA was reversed transcribed and subsequently quantified using microRNA specific Taqman qPCR kits (Thermo Fisher, Waltham, MA, United States). All qPCR reactions were performed in technical triplicates. The $\mathrm{C}_{\mathrm{v}}$ was calculated and 
triplicates resulting in a $\mathrm{C}_{\mathrm{v}}>0.02$ were excluded. MicroRNA expression was normalized to $\mathrm{miR}-191$.

\section{Statistical Analysis}

All statistical analyses, unless otherwise specified, were performed using GraphPad Prism 7 (GraphPad Software, Inc., San Diego, CA, United States). $p$-Values of $<0.05$ were regarded as significant, $p$-values $<0.1$ were regarded as a trend. Normalized relative expression values are presented as mean \pm SEM. Due to large inter-sample variation, Grub's test was used to identify significant $(\alpha<0.05)$ outliers in ncRNA measurements.

One-way analyses of variance (ANOVA) was used to determine differences in expression levels over time within a cohort. To identify significant effects within ANOVAs, the Tukey's Honest Significant Difference-test was used as a post hoc test for multiple comparisons.

When comparing mean expression between different cohorts two-tailed Student's $t$-test were used, paired or unpaired, depending on within or between group comparisons. Values are presented as the fold change between groups.

\section{RESULTS}

\section{Exhaustive Endurance Exercise and Peripheral Arterial Disease}

Samples drawn from five participants of the EEE and five participants of the PAD cohorts were sent to Exiqon in Denmark for multiplex rt/qPCR expression profiling. The multiplex panel measures a total of 179 microRNA, of which, on average, 161 microRNAs were detected per sample. 30 microRNAs were consistently detected in all samples. Although all samples passed quality control, the microRNA content, both in number of detected microRNAs and expression levels of the detected microRNAs, was lowest in PAD cohort samples (Figure 2).

When allowing unsupervised clustering on expression data of the 30 microRNAs expressed in all samples, it is obvious from the heatmap (Figure 3) that all PAD samples cluster together and are clearly different from the athletes' samples. When looking at the athletes' samples, overall clusters are formed, mostly per individual. Inter-individual differences in microRNA profiles are bigger than exercise-induced differences. However, several individual microRNAs were significantly regulated either in response to exhaustive exercise, or in response to recovery (Tables 1A-C).

Table 1A shows two microRNAs that are upregulated directly in response to exhaustive exercise, miR-193a-5p (3.3-fold) and miR-29a-3p (1.3-fold). MiR-29a-3p is the only microRNA that is also regulated after the second exercise session (1.5-fold) (Table 1C), and thus potentially the most reliable biomarker for muscle exhaustion. As shown in Table 2C, multiple microRNAs are regulated in response to a second exercise session. Two microRNAs, miR-99b-5p and miR-151a-3p are downregulated (or depleted) the morning after the first exercise session (-1.6and -1.2 -fold, respectively) (Table 1B), indicating that they may be involved in the recovery process. Unfortunately, these microRNAs did not differ, at any time point, from the PAD samples (Tables $\mathbf{2 A - D}$ ), our pathological model for impaired muscle recovery.

As expected, the number of differentially expressed microRNAs between athletes and PAD patients at any given time point is much larger than between time points within the group of athletes (Tables 2A-D). However, there are several changes in circulating microRNA expression profiles. There are several microRNAs that lose their difference to PAD samples directly after the second exercise session. MiR-193a-5p even loses its significant difference from PAD samples already after

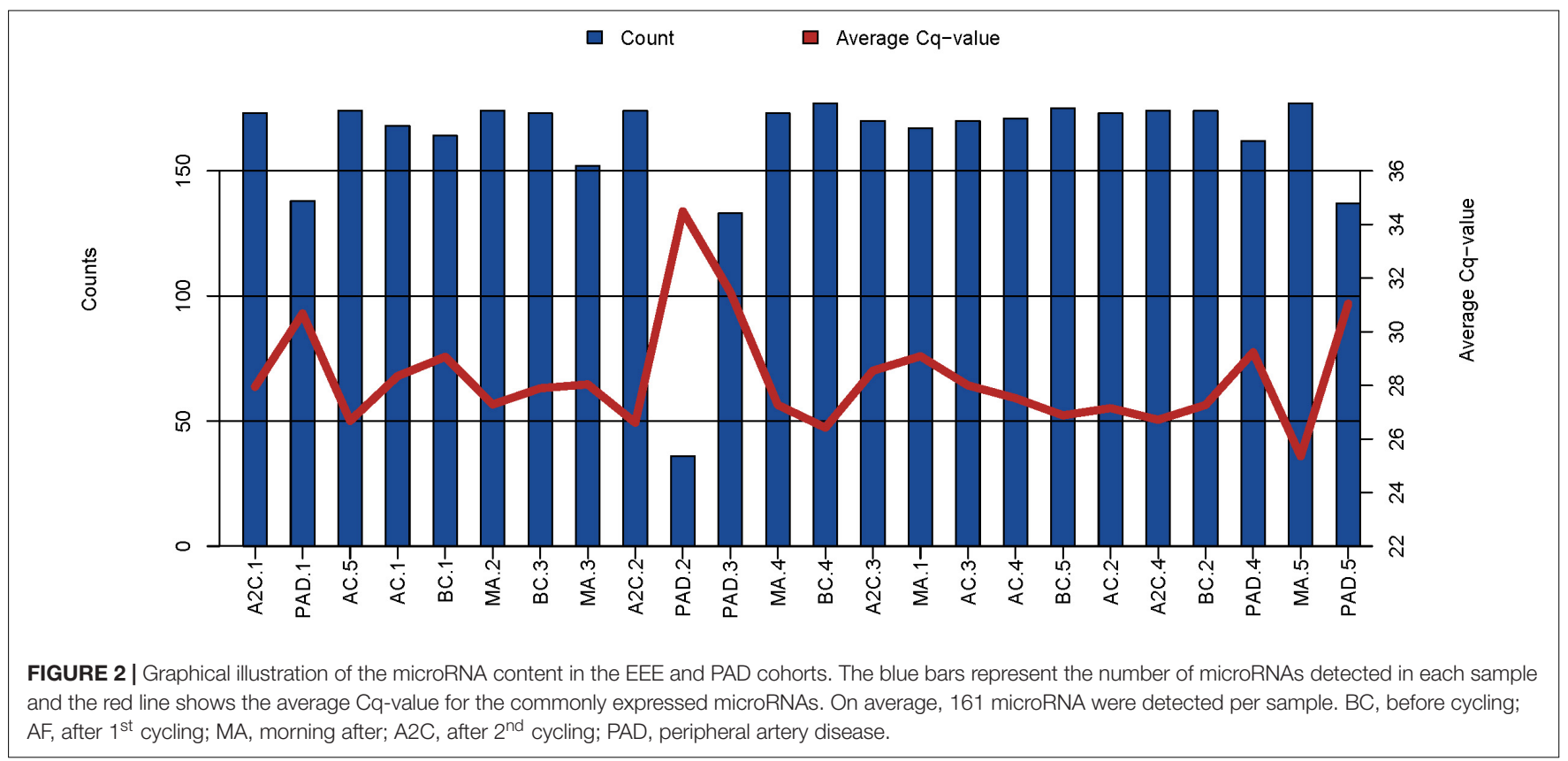



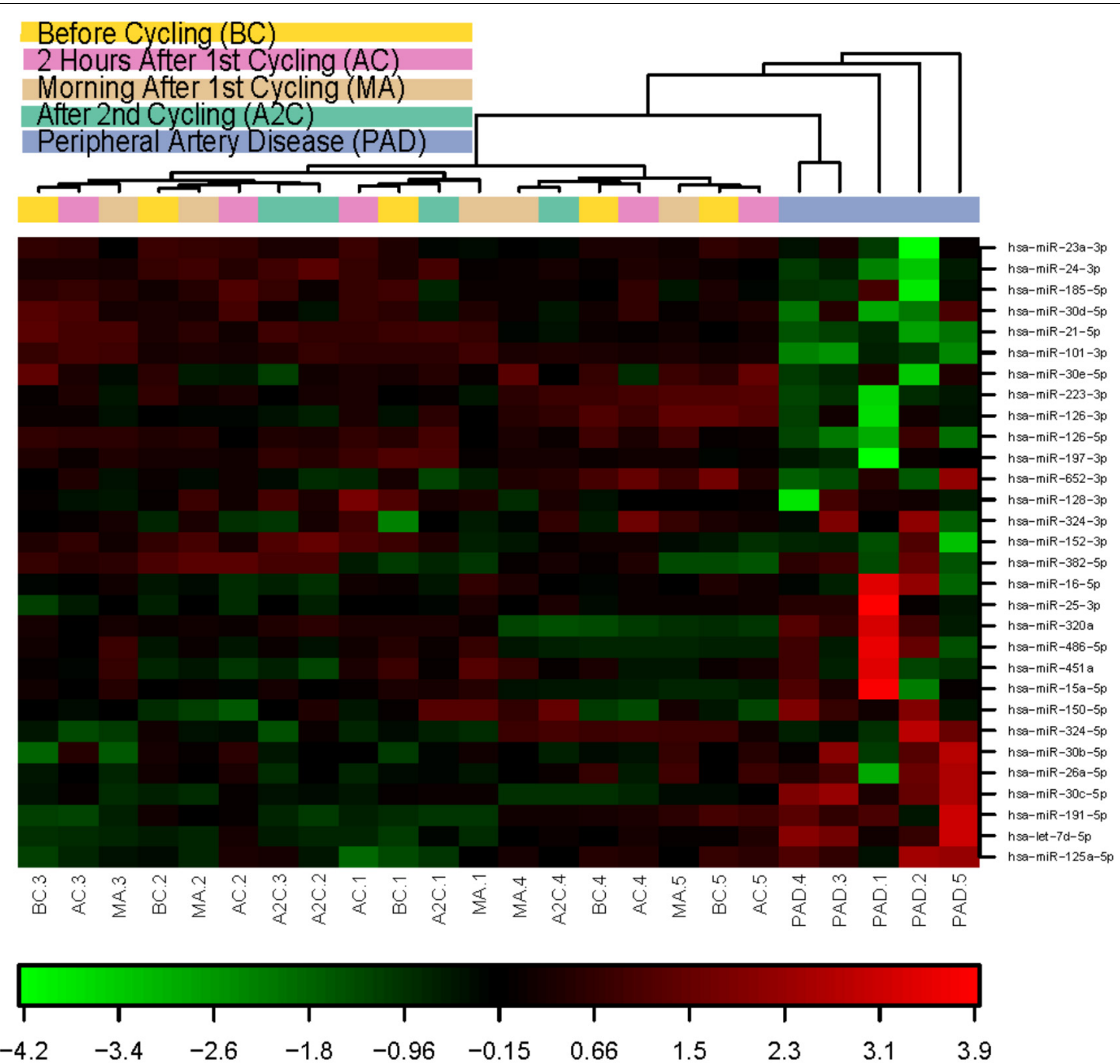

FIGURE 3 | Heat map and unsupervised hierarchical clustering. The clustering was performed on all samples, and on the top 30 microRNAs with highest standard deviation. The normalized expression values $(\mathrm{dCq})$ were used for the analysis. BC, before cycling; AF, after $1^{\text {st }} \mathrm{cycling}$; MA, morning after; $\mathrm{A} 2 \mathrm{C}$, after $2^{\text {nd }}$ cycling; PAD, peripheral artery disease.

the first exercise session. One specific microRNA, miR-495-3p is significantly upregulated in response to both exercise sessions, compared to the PAD samples (3.7- and 4.5-fold, respectively).

The microarray data are available via the Gene Expression Omnibus $\left(\mathrm{GEO}^{1}\right)$ via Accession No. GSE117654.

\section{Time Trial Exercise and Peripheral Arterial Disease}

We selected nine microRNAs to be measured in a second, but different exercise cohort, where elite cyclists were subjected to a strenuous, but non-exhaustive, time trial. MiR-29a-3p, miR-193a-5p, miR-495-3p, miR-106b-5p, and miR-155-3p were selected based on our findings in the EEE cohort. MiR-1-3p, miR133a-3p, miR-133b-3p, and miR-122-5p were selected based on

\footnotetext{
${ }^{1}$ https://www.ncbi.nlm.nih.gov/geo/
}

literature describing their regulation in plasma in response to exercise (Cui et al., 2015; Horak et al., 2016).

MiR-29a-3p and miR-193a-5p were both upregulated at $2 \mathrm{~h}$ after exhaustive exercise (Figure 4; ANOVA $p=0.0019$ and $p=0.0924$, respectively). Two hours after the time trial however, both microRNAs were significantly downregulated. Both miR106b-5p and miR-155-5p were also downregulated after the time trial, but both microRNAs normalized rapidly (Figure 4; ANOVA $p=0.0293$ and $p=0.0058$, respectively).

Of the 'myomiRs', only miR-133b-3p was regulated significantly in response to the time trial, although a similar trend was observed for miR-1-3p (ANOVA $p=0.0179$ and $p=0.0949$, respectively). Both microRNAs appeared upregulated directly after the time trial and decreased in expression during recovery.

When compared to the PAD cohort, we again observed that microRNA expression profiles differ strongly between elite athletes and patients with critical limb ischemia. Most 
TABLE 1 | Regulation of microRNAs in response to exercise.

\begin{tabular}{lcc}
\hline \multicolumn{1}{l}{ MicroRNA } & Fold change & p-Value \\
\hline (A) Two hours after compared to before cycling & \\
hsa-miR-193a-5p & 3.3 & 0.0066 \\
hsa-miR-29a-3p & 1.3 & 0.0081 \\
(B) The morning after compared to before cycling & \\
hsa-miR-99b-5p & -1.6 & 0.0074 \\
hsa-miR-151a-3p & -1.2 & 0.048 \\
(C) After 2 ${ }^{\text {nd }}$ cycling compared to before cycling & \\
hsa-miR-142-3p & 1.3 & 0.0014 \\
hsa-miR-29a-3p & 1.5 & 0.0026 \\
hsa-miR-106b-5p & -1.3 & 0.0058 \\
hsa-miR-141-3p & 1.6 & 0.0082 \\
hsa-miR-30d-5p & -1.2 & 0.011 \\
hsa-miR-150-5p & 1.9 & 0.028 \\
hsa-miR-424-5p & 1.6 & 0.030 \\
hsa-miR-23a-3p & -1.2 & 0.031 \\
hsa-let-7g-5p & 1.3 & 0.033 \\
hsa-miR-423-3p & 1.5 & 0.034 \\
\hline
\end{tabular}

of the microRNAs that we measured, including miR-193a-5p, miR155-5p, miR133a-3p, miR-133b-3p, and miR-122-5p were lower in athletes than in PAD patients at all time points, both before and after exercise. Consistent with the EEE cohort however, miR29a-3p and miR-495-3p only became different from the PAD cohort after exercise. MiR-29a-3p was significantly lower in the TT60 cohort than in the PAD cohort, directly and at $2 \mathrm{~h}$ after the time trial. MiR-495-3p was borderline-significantly lower $(p=0.053)$ in the TT60 cohort than in the PAD cohort in the later stages of recovery, at $5 \mathrm{~h}$ after the time trial.

\section{SnoRNA Expression in the TT60 and PAD Cohorts}

SnoRNAs are also small non-coding RNA molecules that can be reliably measured in human plasma (Liao et al., 2010). As 'snoRnome-wide' screening methods are not yet readily available, we selected a specific subset of snoRNAs and measured these by $\mathrm{rt} / \mathrm{qPCR}$, in order to determine whether or not snoRNAs may be suitable as biomarkers for either exercise or muscle recovery. We selected seven snoRNAs that are expressed from a single gene locus, $14 \mathrm{q} 32$, which also encodes 54 microRNA genes, including miR-495-3p, that we know are involved in angiogenesis and vascular remodeling (Welten et al., 2014, 2017).

All seven snoRNAs could indeed be detected in human plasma. However, especially in the exercise cohort, some snoRNAs were expressed below detection levels in some individual athletes. One snoRNA, SNORD114.1 was significantly upregulated over time after the time trial. SNORD114.1 levels increased gradually throughout the recovery period $(p=0.022)$.

When comparing athletes to PAD patients, the snoRNAs were either different between both cohorts at all TT6o time points (SNORD112, SNORD113.2, SNORD113.6, and SNORD 114.1) or at none or the TT60 time points (SNORD113.7, SNORD113.8, and SNORD113.9). Expression levels of the snoRNAs are presented in Figure 5.
TABLE 2 | Differential expression of microRNAs between elite cyclists and patients with PAD.

\begin{tabular}{lrl}
\hline MicroRNA & Fold change & p-Value \\
\hline (A) Before cycling compared to PAD & -21 & \\
hsa-miR-483-5p & 4.4 & 0.000032 \\
hsa-miR-130a-3p & -2.5 & 0.00031 \\
hsa-miR-2110 & -9.4 & 0.00040 \\
hsa-miR-155-5p & 5.8 & 0.00060 \\
hsa-miR-19a-3p & 3.6 & 0.00075 \\
hsa-miR-21-5p & 4.6 & 0.0015 \\
hsa-miR-19b-3p & -9.5 & 0.0019 \\
hsa-miR-144-5p & 2.9 & 0.0020 \\
hsa-miR-148b-3p & 4.8 & 0.0025 \\
hsa-miR-101-3p & -3.7 & 0.0030 \\
hsa-miR-30c-5p & 5.1 & 0.0035 \\
hsa-miR-590-5p & -4.4 & 0.0037 \\
hsa-let-7a-5p & 8.6 & 0.0044 \\
hsa-miR-27b-3p & -6.1 & 0.0047 \\
hsa-miR-122-5p & -12 & 0.0059 \\
hsa-miR-193a-5p & 4.5 & 0.0064 \\
hsa-miR-335-5p & 2.4 & 0.0082 \\
hsa-miR-106b-5p & -3.5 & 0.0090 \\
hsa-miR-103a-3p & 0.011 \\
hsa-miR-107 & -5 &
\end{tabular}

(B) Two hours after cycling compared to PAD

hsa-miR-130a-3p

hsa-miR-19a-3p

hsa-miR-144-5p

hsa-miR-483-5p

hsa-miR-148b-3p

hsa-miR-21-5p

hsa-miR-19b-3p

hsa-miR-101-3p

hsa-miR-122-5p

hsa-miR-590-5p

hsa-miR-30c-5p

hsa-miR-27b-3p

hsa-miR-155-5p

hsa-let-7a-5p

hsa-miR-335-5p

hsa-miR-103a-3p

hsa-miR-106b-5p

hsa-miR-107

$-2.7$

0.000018
0.00036

0.00063

0.0011

0.0014

0.0020

0.0021

0.0022

0.0023

0.0023

0.0045

0.0052

0.0053

0.0055

0.0060

0.010

0.011

0.012

0.012

hsa-miR-495-3p

(C) The morning after compared to PAD

hsa-miR-122-5p

hsa-miR-130a-3p

hsa-miR-483-5p

hsa-miR-19a-3p

hsa-miR-155-5p

hsa-miR-144-5p

hsa-miR-21-5p

hsa-miR-101-3p

hsa-miR-30c-5p

$$
\begin{array}{r}
-9.8 \\
4.3
\end{array}
$$

$\begin{array}{ll}-9.8 & 0.00044\end{array}$

$4.3 \quad 0.00065$

$-23 \quad 0.00069$

$5.8 \quad 0.00082$

$-5.4 \quad 0.0011$

$\begin{array}{ll}-11 & 0.0017\end{array}$

$3.4 \quad 0.0019$

$5.3 \quad 0.0019$

$\begin{array}{ll}-3.9 & 0.0024\end{array}$ 
TABLE 2 | Continued

\begin{tabular}{|c|c|c|}
\hline MicroRNA & Fold change & $p$-Value \\
\hline hsa-miR-19b-3p & 4.7 & 0.0028 \\
\hline hsa-miR-145-5p & -2.2 & 0.0032 \\
\hline hsa-miR-335-5p & 6.3 & 0.0034 \\
\hline hsa-let-7a-5p & -4.4 & 0.0039 \\
\hline hsa-miR-148b-3p & 2.7 & 0.0039 \\
\hline hsa-miR-27b-3p & 7.7 & 0.0051 \\
\hline hsa-miR-2110 & -3.5 & 0.0059 \\
\hline hsa-miR-590-5p & 5.1 & 0.0069 \\
\hline hsa-miR-885-5p & -11 & 0.0071 \\
\hline hsa-miR-193a-5p & -11 & 0.0079 \\
\hline hsa-miR-103a-3p & -3.7 & 0.0095 \\
\hline \multicolumn{3}{|c|}{ (D) After $2^{\text {nd }}$ cycling compared to PAD } \\
\hline hsa-miR-19a-3p & 5.9 & 0.00023 \\
\hline hsa-miR-19b-3p & 4.8 & 0.00068 \\
\hline hsa-miR-122-5p & -8.8 & 0.0012 \\
\hline hsa-miR-130a-3p & 5.6 & 0.0015 \\
\hline hsa-miR-144-5p & -11 & 0.0015 \\
\hline hsa-miR-148b-3p & 3.0 & 0.0022 \\
\hline hsa-miR-590-5p & 5.8 & 0.0024 \\
\hline hsa-miR-21-5p & 3.6 & 0.0027 \\
\hline hsa-miR-101-3p & 5.2 & 0.0029 \\
\hline hsa-miR-30c-5p & -3.5 & 0.0035 \\
\hline hsa-miR-27b-3p & 8.2 & 0.0048 \\
\hline hsa-miR-483-5p & -21 & 0.0055 \\
\hline hsa-miR-142-5p & 6.9 & 0.0058 \\
\hline hsa-miR-107 & -3.7 & 0.0064 \\
\hline hsa-miR-495-3p & 4.5 & 0.0065 \\
\hline hsa-miR-335-5p & 5.3 & 0.0066 \\
\hline hsa-miR-24-3p & 3.3 & 0.0072 \\
\hline hsa-let-7a-5p & -3.2 & 0.0075 \\
\hline hsa-miR-2110 & -4.1 & 0.0092 \\
\hline hsa-miR-29b-3p & 7.8 & 0.010 \\
\hline
\end{tabular}

\section{DISCUSSION}

In this study, we explored the suitability of circulating small noncoding RNAs, including microRNA and snoRNAs, as biomarkers for exercise and muscle damage. We included two cohorts of elite cyclists, subjected to two different types of exercise regimes, as well as a cohort of patients with PAD that were scheduled to undergo lower limb amputation, due to critical limb ischemia. We identified several microRNAs, as well as one snoRNA, that were regulated in plasma in response to strenuous endurance exercise. Furthermore, we identified microRNAs that may indicate impaired suboptimal muscle repair, as they were only differentially regulated from PAD patients, during the recovery phase in elite cyclists.

The first thing we noticed, when looking at the microRNA profiling data after repeated exhaustive endurance exercise, was the fact that differences in microRNA expression profiles are much bigger between individuals than between the different time points before, during, and after the 2-day exercise regime. Although we did identify several microRNAs that were significantly regulated in response to exercise, our group of athletes was quite homogenous, consisting only of male elite cyclists in their early twenties. MicroRNA expression is highly variable between individuals and is often age-dependent. Therefore, future replication studies in larger and more heterogeneous cohorts are necessary to determine the most reliable microRNA-biomarkers.

We did identify two microRNAs that were significantly regulated in response to the first exhaustive exercise session, miR-29a-3p and miR-193a-5p. MiR-29a-3p was the most consistently regulated, as it was also upregulated on the second day. MiR-29a-3p had also previously been shown to be regulated in response to exercise (de Gonzalo-Calvo et al., 2015). Both microRNAs were also regulated in the second exercise cohort, in response to a $60 \mathrm{~min}$ time trial. However, after non-exhaustive endurance exercise, both miR-29a-3p and miR193a-5p were downregulated in plasma. Although surprising, this is a promising finding, because this could mean that levels of miR$29 a-3 p$ and miR-193a-5p may help distinguish between different exercise regimes and indicate the extent of muscle exhaustion in athletes.

MiR29a-3p, like the other microRNAs of the miR-29-family, plays a crucial role in the formation and maintenance of the extracellular matrix and controls, amongst others, the production of collagens. Less is known about the function of miR-193a, but a recent study has shown that miR-193a interacts with High mobility group box-1 (HMGB1) and has effects on angio- and vasculogenesis (Khoo et al., 2017). HMGB1 is a well-described nuclear protein with a central role in inflammation and apoptosis (Yang et al., 2013). Whether or not miR-29a-3p and miR193-5p play a functional role in muscle physiology, or if they are markers for changes in the musculature remains to be determined.

A third microRNA, miR-106b-5p could potentially provide information about the recovery after exercise. MiR-106b-5p is downregulated in response to both exhaustive and endurance exercise, but only immediately after exercise completion (after the second exercise session) and to the $60 \mathrm{~min}$ time trial. However, $2 \mathrm{~h}$ after the first exhaustive exercise session, and also $2 \mathrm{~h}$ after completion of the $60 \mathrm{~min}$ time trial, miR106b-5p have already normalized to the same levels as before exercise. MiR-106b-5p has previously been shown to be highly expressed in skeletal muscle of diabetic patients (Gallagher et al., 2010) and mouse studies show that miR-106b overexpression result in skeletal muscle mitochondrial dysfunction and insulin resistance (Zhang et al., 2013). Exercise has a well-known effect on insulin resistance and sensitivity (Borghouts and Keizer, 2000) and possibly, miR-106-5p plays a role in this process.

When looking at the more classic myomiRs, only miR-133b$3 p$ and possibly miR-1-3p were regulated in response to exercise after the 60 min time trial. But then again, reproducibility in exercise biomarker studies appears relatively low throughout literature. Many different microRNAs, as well as many different cytokines have been reported to associate with various forms of exercise over the years (Ultimo et al., 2018). One explanation could be that different forms and intensities of exercise can have 

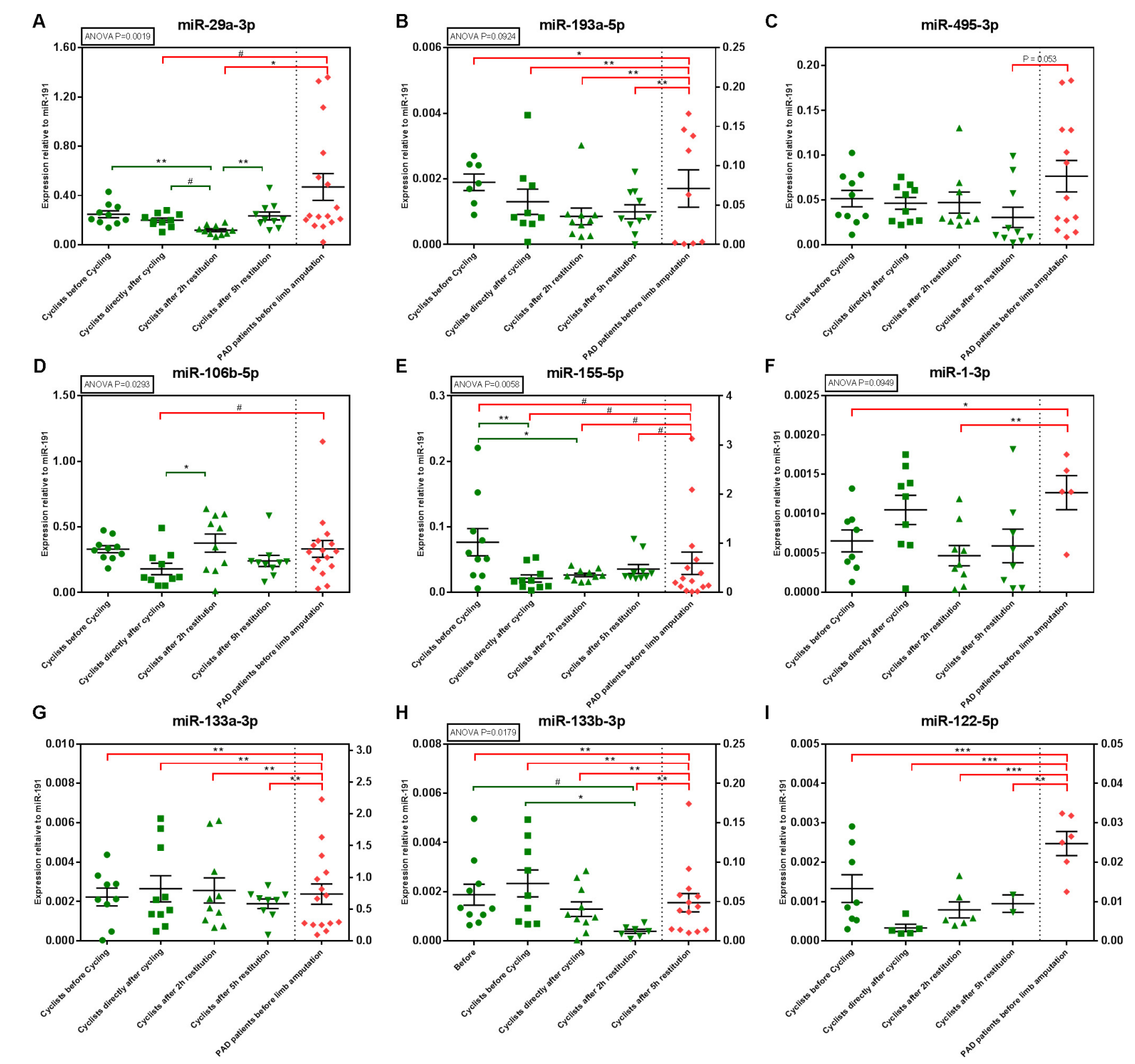

FIGURE 4 | MicroRNA expression in the TT60 and PAD cohorts. Individual sample values are represented by green (athletes) and red (PAD patients) symbols; means with their corresponding SEMs are represented by black lines. ANOVAs were performed to determine circulating microRNA regulation over time and Tukey's Honest Significant Difference-tests were performed to determine differences between time points, within the TT60 cohort ( $N=10$; but not all samples gave detectable microRNA levels at all time points). Differences between the PAD cohort ( $N=16$; but not all samples gave detectable microRNA levels at all time points) and different time points of the TT60 cohort were analyzed using unpaired, two-tailed Student's $t$-tests. \#p $<0.1 ;{ }^{*} p<0.05 ;{ }^{* *} p<0.01 ;{ }^{* * *} p<0.001$. (B,E,G-I) The TT60 and PAD cohort levels are expressed on separate $X$-axes.

profoundly different physiological effects. Another explanation could be that inter-individual differences are simply larger than differences induced by the exercise regimes. Indeed, in our unsupervised clustering of microRNA expression (Figure 3), we observed that microRNAs are clustered by individual, rather than by time point.

A major finding in the present study was that exercise influences plasma snoRNA levels. snoRNAs have previously been described to be stable in the circulation (Liao et al., 2010), however, this is the first report of exercise-mediated regulation of snoRNAs. SnoRNAs may provide more reliable biomarkers in the future. Out of seven snoRNAs, SNORD114.1 is significantly upregulated in response to exercise and increases further during recovery. These seven snoRNAs were selected because they are transcribed from the same gene locus as microRNA miR-495-3p. However, if snoRnome-wide screening methods were to become commercially available, snoRNAs may offer much more information on muscle condition and recovery. 

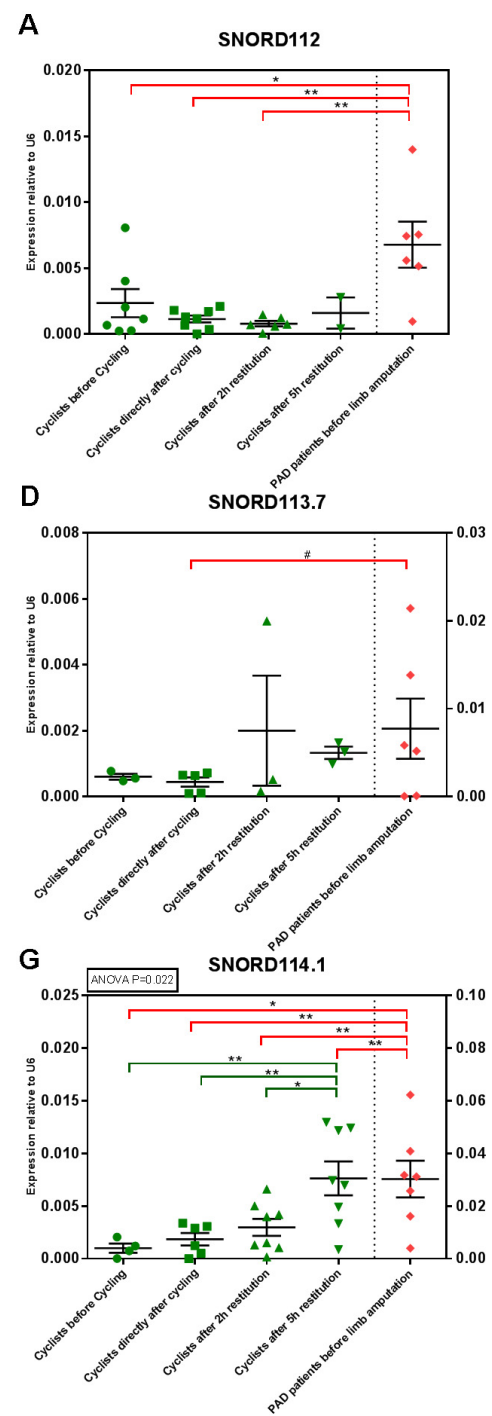

B

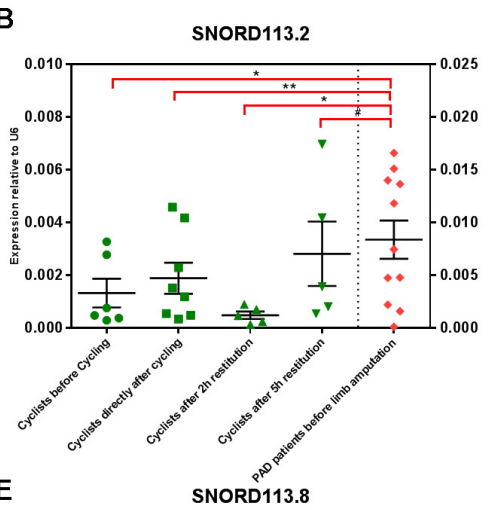

E

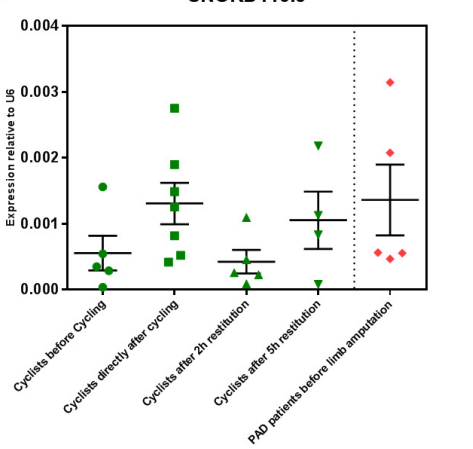

C
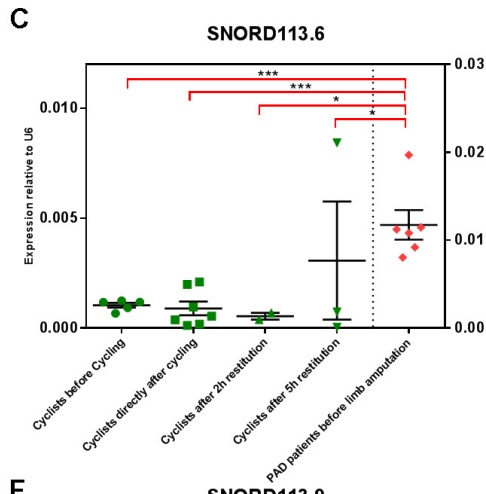

$\mathbf{F}$

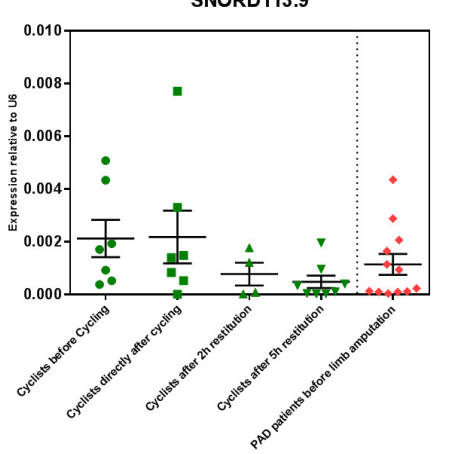

FIGURE 5 | SnoRNA expression in the TT60 and PAD cohorts. Individual sample values are represented by green (athletes) and red (PAD patients) symbols; means with their corresponding SEMs are represented by black lines. ANOVAs were performed to determine circulating snoRNA regulation over time and Tukey's Honest Significant Difference-tests were performed to determine differences between time points, within the $T 60$ cohort $(N=10$; but not all samples gave detectable snoRNA levels at all time points). Differences between the PAD cohort ( $N=16$; but not all samples gave detectable snoRNA levels at all time points) and different time points of the TT60 cohort were analyzed using unpaired, two-tailed Student's $t$-tests. $\# p<0.1 ;{ }^{*} p<0.05 ;{ }^{* *} p<0.01 ;{ }^{* * *} p<0.001$. (B-D,G) The TT60 and PAD cohort levels are expressed on separate $X$-axes.

However, further exploratory studies are certainly necessary to establish circulating snoRNAs as biomarkers for any form of exercise.

Finally, we identified two microRNAs that may function as markers of impaired muscle repair and recovery. Both miR-29a-3p and miR-495-3p are significantly different in athletes compared to PAD patients, but only following exercise. The fact that these microRNAs differ significantly from PAD patients specifically during the recovery stage indicates that plasma levels of these microRNAs may be a marker for muscle repair. Especially miR-495-3p is a promising candidate to mark impaired muscle repair and end-stage PAD, as we have previously shown that miR-495-3p is highly functional in a mouse model for severe PAD (Welten et al., 2014). Inhibition of this microRNA increases endogenous ischemia-induced neovascularization and significantly increased angiogenesis and muscle perfusion. MiR-495-3p is significantly upregulated in response to both exercise sessions, compared to the PAD samples, which indicates that miR-495-3p could be associated with repair processes that are lacking in patients with severe PAD.

Our study design has several limitations. First, we did not include non-exercise control groups in our exercise cohorts. Of course, we measured levels of microRNAs and snoRNAs in all participants before starting the different exercise regimes, but as samples were taken at different time point over 2 days, we could not correct for potential confounding factors like circadian 
rhythm. Similarly, due to our study design where only PAD patients scheduled for limb amputation surgery were included in the study, we did not include samples from PAD patients at other time points. Finally, due to sample depletion, we were unable to confirm the findings from the microarray analyses by qPCR in all the samples of the EEE cohort.

\section{CONCLUSION}

In this study, we have investigated the regulation of circulating small non-coding RNAs in response to exercise. MicroRNAs miR-29a-3p, miR-193a-5p, and miR-106b-5p have shown to be significantly regulated in the circulation in response to endurance exercise. MiR-29a-3p and miR-193a-5p may even be able to distinguish between repeated exhaustive and non-exhaustive exercise. MicroRNAs miR-29a-3p and miR-495-3p may mark impaired muscle recovery in patients with severe critical limb ischemia. Furthermore, we showed for the first time that a circulating snoRNA, SNORD114.1, is regulated in response to endurance exercise.

\section{REFERENCES}

Abul-Khoudoud, O. (2006). Diagnosis and risk assessment of lower extremity peripheral arterial disease. J. Endovasc. Ther. 13(Suppl. 2), II10-II18. doi: $10.1177 / 15266028060130$ S205

Bartel, D. P. (2004). MicroRNAs: genomics, biogenesis, mechanism, and function. Cell 116, 281-297. doi: 10.1016/S0092-8674(04)00045-5

Borghouts, L. B., and Keizer, H. A. (2000). Exercise and insulin sensitivity: a review. Int. J. Sports Med. 21, 1-12. doi: 10.1055/s-2000-8847

Cui, S. F., Li, W., Niu, J., Zhang, C. Y., Chen, X., and Ma, J. Z. (2015). Acute responses of circulating microRNAs to low-volume sprint interval cycling. Front. Physiol. 6:311. doi: 10.3389/fphys.2015.00311

de Gonzalo-Calvo, D., Davalos, A., Montero, A., Garcia-Gonzalez, A., Tyshkovska, I., Gonzalez-Medina, A., et al. (2015). Circulating inflammatory miRNA signature in response to different doses of aerobic exercise. J. Appl. Physiol. 119, 124-134. doi: 10.1152/japplphysiol.00077. 2015

Gallagher, I. J., Scheele, C., Keller, P., Nielsen, A. R., Remenyi, J., Fischer, C. P., et al. (2010). Integration of microRNA changes in vivo identifies novel molecular features of muscle insulin resistance in type 2 diabetes. Genome Med. 2:9. doi: $10.1186 / g m 130$

Germani, A., Di Campli, C., Pompilio, G., Biglioli, P., and Capogrossi, M. C. (2009). Regenerative therapy in peripheral artery disease. Cardiovasc. Ther 27, 289-304. doi: 10.1111/j.1755-5922.2009.00105.x

Hakimzadeh, N., Nossent, A. Y., Van Der Laan, A. M., Schirmer, S. H., De Ronde, M. W., Pinto-Sietsma, S. J., et al. (2015). Circulating micrornas characterizing patients with insufficient coronary collateral artery function. PLoS One 10:e0137035. doi: 10.1371/journal.pone.0137035

Horak, M., Novak, J., and Bienertova-Vasku, J. (2016). Muscle-specific microRNAs in skeletal muscle development. Dev. Biol. 410, 1-13. doi: 10.1016/j.ydbio.2015. 12.013

Khoo, C. P., Roubelakis, M. G., Schrader, J. B., Tsaknakis, G., Konietzny, R., Kessler, B., et al. (2017). miR-193a-3p interaction with HMGB1 downregulates human endothelial cell proliferation and migration. Sci. Rep. 7:44137. doi: $10.1038 /$ srep 44137

Kiss, T. (2001). Small nucleolar RNA-guided post-transcriptional modification of cellular RNAs. EMBO J. 20, 3617-3622. doi: 10.1093/emboj/20.14. 3617

Langleite, T. M., Jensen, J., Norheim, F., Gulseth, H. L., Tangen, D. S., Kolnes, K. J., et al. (2016). Insulin sensitivity, body composition and adipose depots following $12 \mathrm{w}$ combined endurance and strength training in dysglycemic and

\section{AUTHOR CONTRIBUTIONS}

$\mathrm{KH}$ performed the experiments, analyzed the data, and wrote the manuscript. OS performed the experiments, collected the samples, and edited the manuscript. KS collected the Ampubase biobank samples and edited the manuscript. PQ supervised the Ampubase biobank samples collection and edited the manuscript. JJ designed the protocols, supervised the experiments, and edited the manuscript. AN designed the protocols, supervised the experiments, and wrote the manuscript.

\section{FUNDING}

This study was supported by grants from the Dutch Heart Foundation (2014T102 to AN) and the Danish Council for Independent Research - DFF Medical Sciences (6120-00049B to $\mathrm{KH}$ ). The exercise physiology studies were partly supported by Arla Foods Ingredients Group P/S and Team Denmark (Organization funded by the Danish Government for promotion of elite sports).

normoglycemic sedentary men. Arch. Physiol. Biochem. 122, 167-179. doi: 10. 1080/13813455.2016.1202985

Liao, J., Yu, L., Mei, Y., Guarnera, M., Shen, J., Li, R., et al. (2010). Small nucleolar RNA signatures as biomarkers for non-small-cell lung cancer. Mol. Cancer 9:198. doi: 10.1186/1476-4598-9-198

Mitchell, P. S., Parkin, R. K., Kroh, E. M., Fritz, B. R., Wyman, S. K., PogosovaAgadjanyan, E. L., et al. (2008). Circulating microRNAs as stable blood-based markers for cancer detection. Proc. Natl. Acad. Sci. U.S.A 105, 10513-10518. doi: 10.1073/pnas.0804549105

Peiro-Chova, L., Pena-Chilet, M., Lopez-Guerrero, J. A., Garcia-Gimenez, J. L., Alonso-Yuste, E., Burgues, O., et al. (2013). High stability of microRNAs in tissue samples of compromised quality. Virchows Arch. 463, 765-774. doi: 10. 1007/s00428-013-1485-2

Rustad, P. I., Sailer, M., Cumming, K. T., Jeppesen, P. B., Kolnes, K. J., Sollie, O., et al. (2016). Intake of protein plus carbohydrate during the first two hours after exhaustive cycling improves performance the following day. PLoS One 11:e0153229. doi: 10.1371/journal.pone.0153229

Ultimo, S., Zauli, G., Martelli, A. M., Vitale, M., Mccubrey, J. A., Capitani, S., et al. (2018). Influence of physical exercise on microRNAs in skeletal muscle regeneration, aging and diseases. Oncotarget 9, 17220-17237. doi: 10.18632/ oncotarget. 24991

Ulven, S. M., Foss, S. S., Skjolsvik, A. M., Stadheim, H. K., Myhrstad, M. C., Raael, E., et al. (2015). An acute bout of exercise modulate the inflammatory response in peripheral blood mononuclear cells in healthy young men. Arch. Physiol. Biochem. 121, 41-49. doi: 10.3109/13813455.2014.10 03566

Welten, S. M., Bastiaansen, A. J., De, J. R., De Vries, M. R., Peters, E. H., Boonstra, M., et al. (2014). Inhibition of 14q32 MicroRNAs miR-329, miR-487b, miR-494 and miR-495 increases neovascularization and blood flow recovery after ischemia. Circ. Res. 115, 696-708. doi: 10.1161/CIRCRESAHA.114.30 4747

Welten, S. M. J., De Jong, R. C. M., Wezel, A., De Vries, M. R., Boonstra, M. C., Parma, L., et al. (2017). Inhibition of 14q32 microRNA miR-495 reduces lesion formation, intimal hyperplasia and plasma cholesterol levels in experimental restenosis. Atherosclerosis 261, 26-36. doi: 10.1016/j.atherosclerosis.2017. 04.011

Whitham, M., Parker, B. L., Friedrichsen, M., Hingst, J. R., Hjorth, M., Hughes, W. E., et al. (2018). Extracellular vesicles provide a means for tissue crosstalk during exercise. Cell Metab. 27, 237.e4-251.e4. doi: 10.1016/j.cmet.2017.12.001

Wilhelm, E. N., Gonzalez-Alonso, J., Parris, C., and Rakobowchuk, M. (2016). Exercise intensity modulates the appearance of circulating microvesicles 
with proangiogenic potential upon endothelial cells. Am. J. Physiol. Heart Circ. Physiol. 311, H1297-H1310. doi: 10.1152/ajpheart.00516. 2016

Yang, H., Antoine, D. J., Andersson, U., and Tracey, K. J. (2013). The many faces of HMGB1: molecular structure-functional activity in inflammation, apoptosis, and chemotaxis. J. Leukoc. Biol. 93, 865-873. doi: 10.1189/jlb.12 12662

Zhang, Y., Yang, L., Gao, Y. F., Fan, Z. M., Cai, X. Y., Liu, M. Y., et al. (2013). MicroRNA-106b induces mitochondrial dysfunction and insulin resistance in C2C12 myotubes by targeting mitofusin-2. Mol. Cell. Endocrinol. 381, 230-240. doi: 10.1016/j.mce.2013.08.004
Conflict of Interest Statement: The authors declare that the research was conducted in the absence of any commercial or financial relationships that could be construed as a potential conflict of interest.

Copyright $\odot 2018$ Håkansson, Sollie, Simons, Quax, Jensen and Nossent. This is an open-access article distributed under the terms of the Creative Commons Attribution License (CC BY). The use, distribution or reproduction in other forums is permitted, provided the original author(s) and the copyright owner(s) are credited and that the original publication in this journal is cited, in accordance with accepted academic practice. No use, distribution or reproduction is permitted which does not comply with these terms. 